\title{
Simulation and Optimization for Railway Operations Management
}

\author{
Andrea D'Ariano ${ }^{D},{ }^{1}$ Francesco Corman, ${ }^{2}$ Taku Fujiyama, ${ }^{3}$ \\ Lingyun Meng, ${ }^{4}$ and Paola Pellegrini $\oplus^{5}$ \\ ${ }^{1}$ Roma Tre University, Rome, Italy \\ ${ }^{2}$ ETH Zurich, Zurich, Switzerland \\ ${ }^{3}$ University College London, London, UK \\ ${ }^{4}$ Beijing Jiaotong University, Beijing, China \\ ${ }^{5}$ Institut Français des Sciences et Technologies des Transports, de l'Aménagement et des Réseaux, Villeneuve d'Ascq Cedex, France
}

Correspondence should be addressed to Andrea D’Ariano; dariano@ing.uniroma3.it

Received 26 June 2018; Accepted 26 June 2018; Published 2 August 2018

Copyright (C) 2018 Andrea D’Ariano et al. This is an open access article distributed under the Creative Commons Attribution License, which permits unrestricted use, distribution, and reproduction in any medium, provided the original work is properly cited.

In the forthcoming decades, railway transport is expected to face a significant growth of traffic flows which will mostly have to be accommodated over the existing infrastructures. An increase of capacity utilization is needed to avoid reduction of reliability and punctuality of transport services. Furthermore, energy-efficient operations and multimodality opportunities are also topics of growing interest.

Intelligent railway operations management requires accurate modelling and simulation of train and pedestrian traffic flows and optimal management of key decisions at strategic, tactical, and operational levels. This special issue focuses on advanced mathematical modelling and optimal control in the railway domain and related multimodal transport networks. We aim to identify new methods for improving the effectiveness and efficiency of railway operations, including the development of advanced algorithmic techniques for timetabling, capacity management, infrastructure management, and traffic and passengers flow management.

This special issue has selected a compendium of research papers addressing recent theoretical and practical advances on railway operations management. 32 papers were submitted to this special issue, 14 of which were accepted for publication. As the guest editors of this special issue, we next summarize the 14 accepted papers.

In the paper titled "A Short Turning Strategy for Train Scheduling Optimization in an Urban Rail Transit Line: The
Case of Beijing Subway Line 4" by M. Zhang et al., the authors propose a mixed-integer nonlinear programming model for the train scheduling with consideration of short turning strategy and train circulation plan. Mixed-integer linear programming approach is used to solve this optimization problem. Two case studies are carried out based on the data of Beijing subway line 4 . The simulation results show that operation pattern with short turning train services can acquire a better train schedule to meet passenger demand and a better train circulation plan.

In the paper titled "Integrated Optimization on Train Control and Timetable to Minimize Net Energy Consumption of Metro Lines," Y. Zhou et al. present an integrated optimization model on train control and timetable to minimize the net energy consumption with consideration of utilizing regenerative energy. An improved model and algorithms on train control are proposed to attain energy-efficient speed profiles. Case studies on Beijing Metro Line 5 illustrate that the improved train control approach can save traction energy consumption by $20 \%$ in comparison with the commonly adopted train control sequence in timetable optimization.

In the paper titled "Application of Data Clustering to Railway Delay Pattern Recognition" by F. Cerreto et al., the authors employ $K$-means clustering to identify recurrent delay patterns on a high traffic railway line north of Copenhagen, Denmark. The clusters identify behavioral patterns 
in the very large ("big data") data sets generated automatically and continuously by the railway signal system. The results reveal where corrective actions are necessary, showing where recurrent delay patterns take place. The demonstrated methodology is scalable and can be potentially transferred to any system of transport.

In the paper titled "Defining Reserve Times for Metro Systems: An Analytical Approach" by L. D’Acierno et al., the authors provide an analytical approach for determining operational parameters for metro systems so as to support the planning and implementation of energy-saving strategies. They develop a suitable methodology for estimating reserve times that represent the main rate of extra time needed to put eco-driving strategies in place. The approach proposed by the authors is applied in Line 1 of the Naples metro system, whose service frequency was duly taken into account, to analyze operation configurations and to quantify the amount of saved energy.

In the paper titled "Using Smart Card Data Trimmed by Train Schedule to Analyze Metro Passenger Route Choice with Synchronous Clustering," W. Li et al. analyze smart card data in Shanghai metro systems to understand mobility patterns. To do that, they cluster travelers and smart card data transactions by looking at the pure travel time. This is then appropriately converted to a model of route choice throughout the network. They found out that those steps can improve substantially the amount of insight into the travelers' behavior.

In "A Simulation Platform for Combined Rail/Road Transport in Multiyards Intermodal Terminals," X. Chen et al. tackle multimodal freight terminals where the combination between road and rail transport is investigated by means of simulation in terminals featuring multiple rail yards. The key features of the proposed approach based on Time Petri Nets are an increased realism of train operations and container movements in Qianchang railway terminal, including train routing dispatching rules. The validation on historical data allows evaluating influence of design parameters on container operations.

In the paper titled "On Individual Repositioning Distance along Platform during Train Waiting," F. Leurent and X. Xie propose a stochastic model in which user's journey is decomposed into phases of, successively, walking in the access station, platform positioning, waiting for boarding, train riding, and walking in the egress station. Egress times and exit instants are random variables that are characterized by distribution and mass probability functions under closed form for both single and distributed walking speed. Maximum likelihood estimation is proposed and applied to a case study of commuter rail line in Paris, France, with satisfactory numerical results.

In the paper titled "Efficiency Assessment of TransitOriented Development by Data Envelopment Analysis: Case Study on the Den-en Toshi Line in Japan," J. Guo et al. assess the efficiency of Transit-Oriented Development (TOD), that is, an urban planning approach that encourages a modal shift from private to public transportation, by applying the Data Envelopment Analysis (DEA) method. The ridership of public transportation is considered as the direct output characteristic of TOD efficiency, and nine indicators of ridership are selected as inputs of TOD. The Tokyo Den-en Toshi Line in Japan is investigated as a typical case of TOD.

In the paper titled "Stop Plan of Express and Local Train for Regional Rail Transit Line" by Q. Luo et al., the Logit model is used to analyze the behavior of passengers choosing trains by considering the sensitivity of travel time and travel distance. Based on the composition of passenger travel time, an integer programming optimization model for train stop scheme is proposed, which aims at minimizing the total passenger travel time for a certain regional rail line in Shenzhen. Genetic Algorithms are used to solve the problem. The simulation result shows the feasibility of the proposed model and the efficiency of the proposed algorithm.

In the paper titled "Fuzzy Approach in Rail Track Degradation Prediction" by M. Karimpour et al., an adaptive network-based fuzzy inference system (ANFIS) model is proposed to estimate rail track degradation for the curves and straight sections of Melbourne tram track system. A fuzzy approach is proposed due to the nonlinear and noisy nature of the data according to the data that were available on the Melbourne tram network. Experimental results demonstrate that the developed model is capable of estimating the long-term behavior of rail tracks and predicting the gauge values with an R2 of 0.6 and 0.78 for curves and straights, respectively.

In the paper titled "PULSim: User-Based Adaptable Simulation Tool for Railway Planning and Operations," Y. Cui et al. introduce a user-based, customizable platform to provide the ability of defining sophisticated workflows for users. As the preconditions of the platform, the design aspects for modelling the components of a German railway system and building the workflow of railway simulation are elaborated. Based on the model and the workflow, an integrated simulation platform with open interfaces is developed. Users and researchers gain the ability to rapidly develop their own algorithms, supported by the tailored simulation process in a flexible manner.

In the paper titled "The Planners' Perspective on Train Timetable Errors in Sweden" by C.-W. Palmqvist et al., typical errors in train timetables of railways, relevant reasons, and potential benefits of new tools and processes are investigated and reported through reviewing the state of practice and the state of the art in timetable planning, studying the research literature and railway management documents from several European countries, and conducting interviews with timetable planners in Southern Sweden.

In the paper titled "Calculation Method for Load Capacity of Urban Rail Transit Station Considering Cascading Failure," J. Huang et al. quantify the capacity of urban rail transit stations in terms of number of passengers. Their paper relies on an association network of facilities, set up based on the analysis of passenger service chain in station. The analysis of a case study of Lujiabang Road Station in Shanghai shows that this algorithm can search for capacity bottlenecks and help trace the load variation of facilities in different scenarios, providing support for passenger flow organization.

In the paper titled "Optimal Operation of High-Speed Trains Using Hybrid Model Predictive Control" by Y. Yang et al., a control framework is proposed for the controller 
design of an automatic train operation (ATO) system. Firstly, a piecewise linear system is constructed through piecewise linearization of the Chinese high-speed train's nonlinear dynamics. Secondly, the piecewise linear system is transformed into a mixed logical dynamical system. For the latter system, a hybrid model predictive controller is designed to realize the precise control. Simulation results validate the effectiveness of the framework.

Taken together, these 14 papers point to interesting research directions in mathematical modeling, simulation, and particularly optimization to tackle practically oriented railway operations management challenges in several countries worldwide, including China, Denmark, Italy, France, Japan, Australia, Germany, and Sweden.

Andrea D'Ariano Francesco Corman Taku Fujiyama Lingyun Meng Paola Pellegrini 


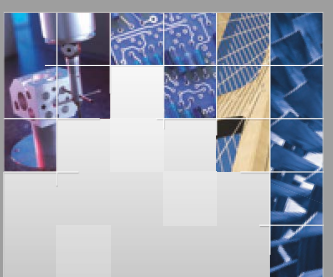

\section{Enfincering}
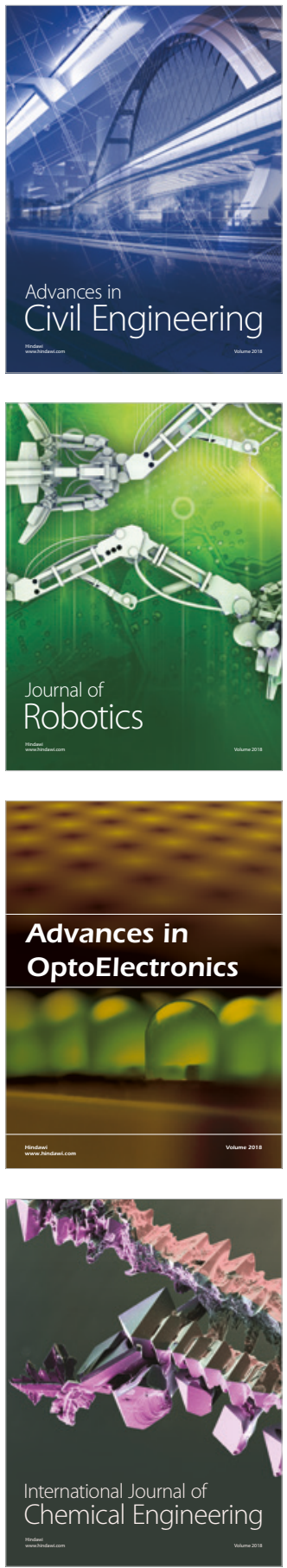

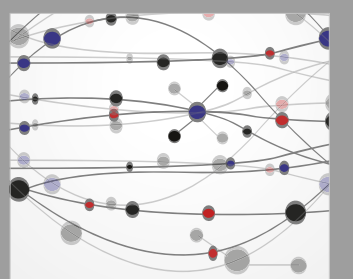

\section{Rotating \\ Machinery}

The Scientific World Journal

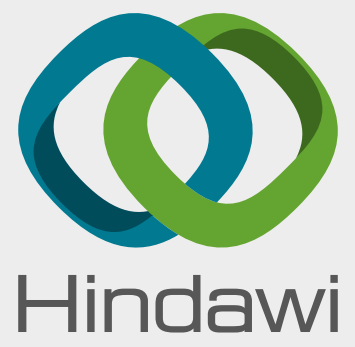

Submit your manuscripts at

www.hindawi.com
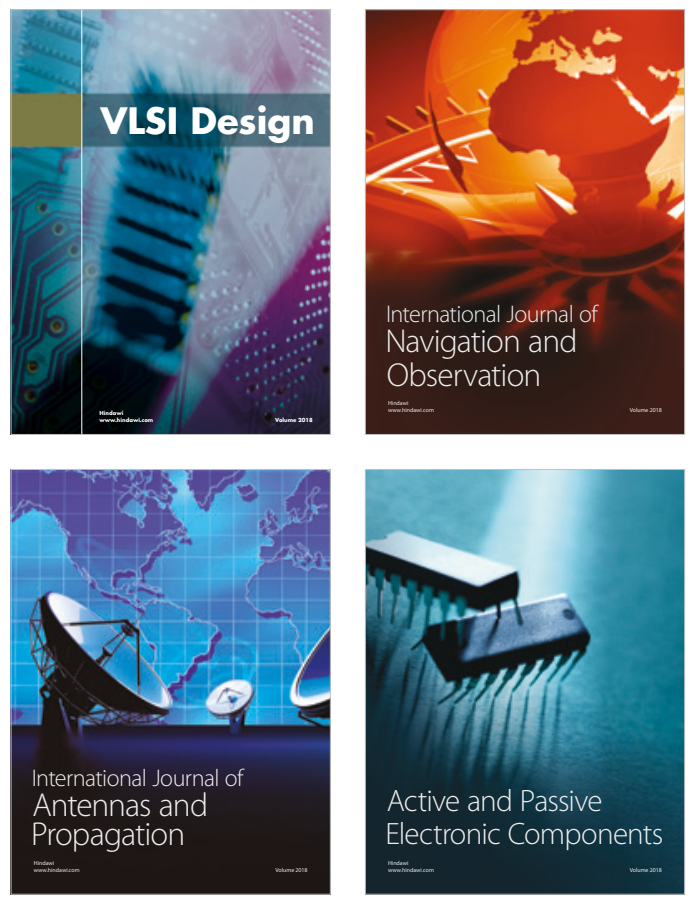
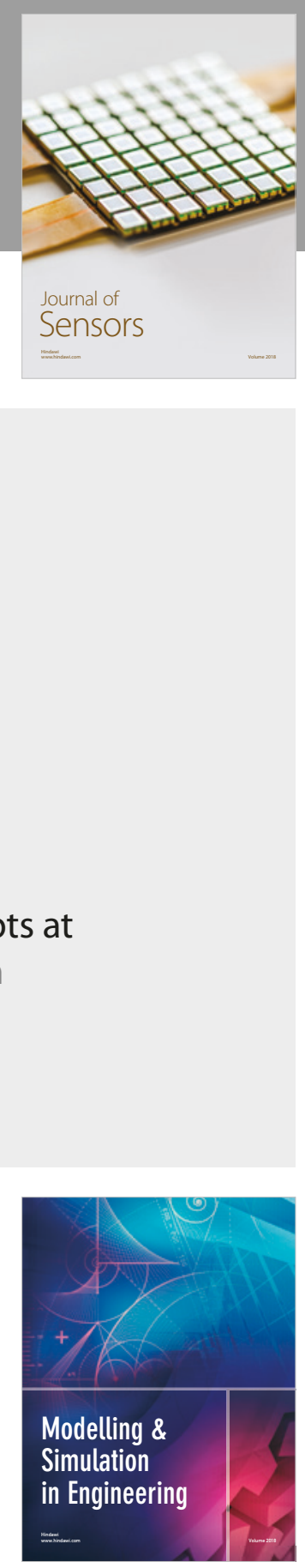

\section{Advances \\ Multimedia}
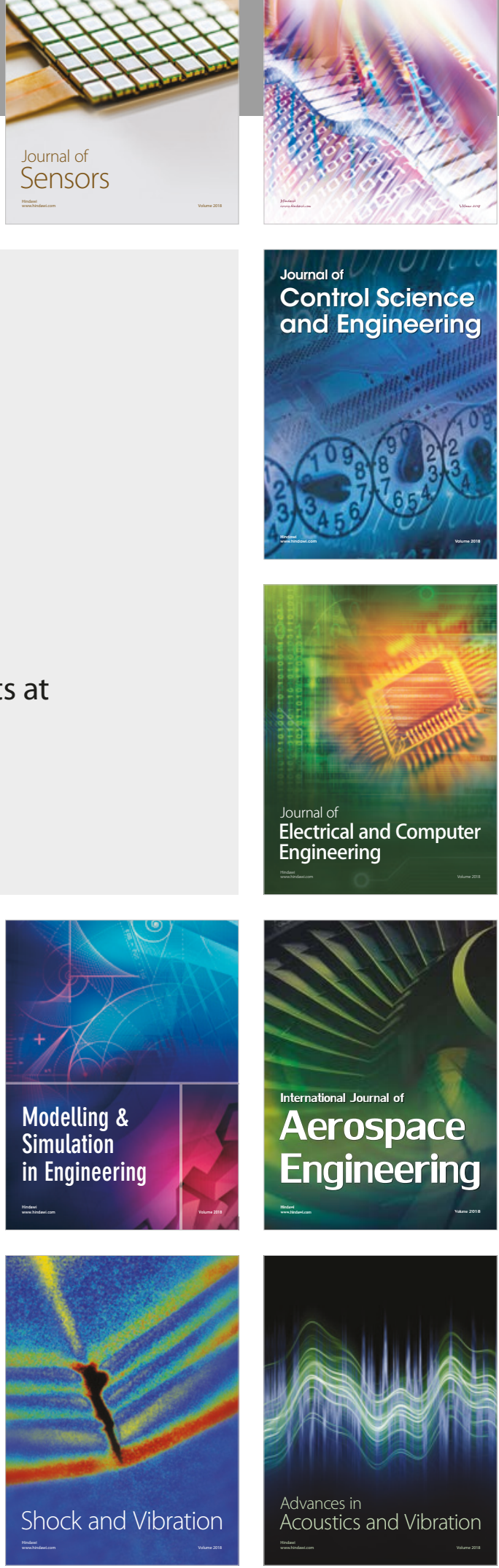